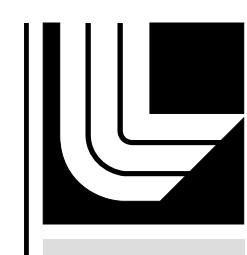

LAWRENCE LIVERMORE N A T IO N A L LABORATORY

\title{
Magnetic Reconnection in the Spheromak: Physics and Consequences
}

E. B. Hooper, B. I. Cohen, D. N. Hill, L. L. LoDestro, H. S. McLean, C. A. Romero-Talamas, R. D. Wood, C. R. Sovinec

March 1, 2006

Journal of Fusion Energy 
This document was prepared as an account of work sponsored by an agency of the United States Government. Neither the United States Government nor the University of California nor any of their employees, makes any warranty, express or implied, or assumes any legal liability or responsibility for the accuracy, completeness, or usefulness of any information, apparatus, product, or process disclosed, or represents that its use would not infringe privately owned rights. Reference herein to any specific commercial product, process, or service by trade name, trademark, manufacturer, or otherwise, does not necessarily constitute or imply its endorsement, recommendation, or favoring by the United States Government or the University of California. The views and opinions of authors expressed herein do not necessarily state or reflect those of the United States Government or the University of California, and shall not be used for advertising or product endorsement purposes. 


\title{
Magnetic Reconnection in the Spheromak: Physics and Consequences*
}

\author{
E. B. Hooper, B. I. Cohen, D. N. Hill, L. L. LoDestro, H. S. McLean, C. A. Romero- \\ Talamás, R. D. Wood \\ Lawrence Livermore National Laboratory \\ 7000 East Avenue, Livermore, CA 94550 \\ C. R. Sovinec \\ University of Wisconsin \\ 1500 Engineering Drive, Madison WI 53706
}

\begin{abstract}
Magnetic reconnection in the spheromak changes magnetic topology by conversion of injected toroidal flux into poloidal flux and by magnetic surface closure (or opening) in a slowly decaying spheromak. Results from the Sustained Spheromak Physics Experiment, SSPX, are compared with resistive MHD simulations using the NIMROD code. Voltage spikes on the SSPX gun during spheromak formation are interpreted as reconnection across a negative-current layer close to the mean-field x-point. Field lines are chaotic during these events, resulting in rapid electron energy loss to the walls and the low $T_{e}<50 \mathrm{eV}$ seen in experiment and simulation during strong helicity injection. Closure of flux sufaces (and high $T_{e}$ ) can occur between voltage spikes if they are sufficiently far apart in time; these topology changes are not reflected in the impedance of the axisymmetric gun. Possible future experimental scenarios in SSPX are examined in the presence of the constraints imposed by reconnection physics.
\end{abstract}

Achievements in the Sustained Spheromak Physics Experiment ${ }^{1}$ include peak electron temperatures of $350 \mathrm{eV}$, edge magnetic fluctuation levels less than $1 \%$, and core thermal diffusivities $<10 \mathrm{~m}^{2} / \mathrm{ss}^{2,3}$ Confinement is independent of the mass (proton or deuteron). ${ }^{4}$ The spectrum of magnetic fluctuations in the core of the spheromak correlates well with the qprofile, with good energy confinement obtained when low-order rational surfaces are absent in the spheromak, ${ }^{3}$ as discussed further by McLean in this issue. ${ }^{5}$ Helicity balance in the driven spheromak has been examined, and the role of losses to resistivity (especially on open field lines) and to sheath effects at the gun cathode and flux conserver clarified. ${ }^{6}$ Reference 6 also demonstrated the experimental importance of magnetic fluctuations for the transport of helicity into the core of the mean-field (azimuthally-averaged) spheromak.

SSPX and resistive MHD simulations using the NIMROD code in realistic geometry have been found to agree in magnetic features at least semiquantitatively and in many cases quantitatively. ${ }^{7,8}$ The simulations have also been used to examine the physics of magnetic reconnection in the conversion of toroidal flux injected from the gun into poloidal flux within the spheromak. ${ }^{9}$ These reconnection simulations will be compared more fully with experiment in this paper, and they will be used to consider some possible future opportunities for the experiment.

Figure 1 shows two experimental discharges with characteristics typical of SSPX. Shown in both are the gun current, voltage, and the magnetic field at the flux conserver midplane. The current is also plotted in terms of the normalized quantity, $\lambda_{\text {gun }}=\mu_{0} I_{\text {gun }} / \Psi_{\text {gun }}$,

* Work performed under the auspices of the U. S. DOE by U. California LLNL under contract No. W-7405-Eng-48. 
where $I_{g u n}$ is the current injected from the gun and $\Psi_{\text {gun }}$ is the gun flux. A similar ratio, defined for force-free plasmas as $\lambda=\mu_{0} \mathbf{j} \cdot \mathbf{B} / B^{2}$, yields Ampere's law in the form $\nabla \times \mathbf{B}=\lambda \mathbf{B}$. If this is solved as an eigenvalue problem in the SSPX flux conserver, the lowest eigenvalue is about $9.6 \mathrm{~m}^{-1}$. It is known that $\lambda_{\text {gun }}$ must be greater than this value to form a spheromak, c.f. the "downhill in $\lambda$ " rule-of-thumb. ${ }^{10}$ A series of NIMROD simulations, run to determine the poloidal flux amplifcation in SSPX as a function of $\lambda_{\text {gun }}$, are shown in Fig. 2. Below $10 \mathrm{~m}^{-1}$ the flux amplification is 1 (no amplification); this is the threshold, $\lambda_{T H}$, which is plotted as dashed lines in Fig. 1.

We note that the gun voltage for the steadily-building spheromak has strong spikes. ${ }^{11}$ We see in Fig. 3 that strong spikes are also present during the high-current phase of the pulsed-formation discharge. Simulations below indicate that these spikes are associated with magnetic reconnection events in the plasma. These convert toroidal flux into poloidal flux, and the voltage spikes may be thought of as a change in the discharge inductance during each reconnection event. The smaller voltage spikes during the low-current phase of the pulse-formation discharge are not present in simulations and may be due to an instability on the surface of the gun cathode. ${ }^{12}$

Figure 4 shows a pair of spikes from a simulation which demonstrates the increase in the spheromak poloidal magnetic field which they generate. Simultaneously with each spike the poloidal flux increases showing the association with magnetic-reconnection events. The amplitude of the $n=1$ mode drops, with the next event occuring when the mode energy has grown to a level comparable to that in the first event. Between events, the electron energy increases due to ohmic heating, droping rapidly when the event creates magnetic stochasticity and field lines reach the electrodes (Fig. 5). The same event which allows current and helicity to penetrate into the spheromak has also greatly reduced the energy confinement time.

The simulation shows current sheets associated with the $n=1$ mode, as seen in Fig. 6 . These current sheets have a thickness consistent with resistive magnetic reconnection across the sheet in times comparable to the pulse times seen in the simulation and experiment; see details in Ref. 9. Romero-Talamás ${ }^{13}$ is presently making magnetic probe measurements in the interior of SSPX during the spheromak formation phase, and comparing these to three dimensional renderings of the simulation results. The goal of this work will be to determine the validity of the simulation simulations and to extend our understanding of the physics involved in forming a spheromak.

In simulations to date, operation at sufficiently high gun current to build or sustain a steady-state spheromak generates open magnetic field lines. This is consistent with experiments in SSPX, although open field lines cannot be directly diagnosed. If this result continues to hold, it will be important to control separately the plasma buildup and confinement phases. A possible scenario is to build the plasma with $\lambda_{\text {gun }}>\lambda_{T H}$; during this time the field lines are likely stochastic and temperatures low. The confinement phase with $\lambda_{\text {gun }}<\lambda_{T H}$ would have a slow decay of the magnetic field energy until a rebuild current-pulse is applied; this pulse would retain considerable magnetic energy and not have to form the basic spheromak. A reactor based on this scenario would have a burn phase, followed by a dump of ash and a rebuilding of the plasma for a new burn phase. Initial experiments have demonstrated the feasibility of this scenario, ${ }^{14,15}$ and simulations using NIMROD are underway. Future experiments will use a new modular capacitor bank which is being installed to allow higher gun currents, longer pulses, and considerable flexibility in the pulse shapes. 
Plasma optimization may be significantly improved if heating is enhanced over that from ohmic curent alone. The ohmic heating inside the separatrix of the mean-field spheromak is several MW, so significant additional heating can be supplied by neutral beams. This heating would also allow energy transport to be better separated from magnetic field generation, allowing a fuller understanding of spheromak physics. Pulsed beams are planned for SSPX, with physics studies and installation design underway.

Energy confinement and its relation to interior magnetic modes generated at low-order rational surfaces ${ }^{3}$ also motivate the study of control of the spheromak. A new, modular capacitor bank may provide a tool for this control; simulation studies are also being done to address this possibility.

\section{References}

1. E. B. Hooper, L. D. Pearlstein, and R. H. Bulmer, Nucl. Fusion 39, 863 (1999).

2. R. D. Wood, D. N. Hill, E. B. Hooper, S. Woodruff, H. S. McLean, and B. W. Stallard, Nucl. Fusion 45, 1582 (2005).

3. H. S. McLean, R. D. Wood, B. I. Cohen, E. B. Hooper, D. N. Hill, J. M. Moller, C. Romero-Talamás, S. Woodruff, Phys. Plasmas (to be published).

4. R. D. Wood, D. N. Hill, E. B. Hooper, H. S. McLean, D. Ryutov, and S. Woodruff, J. Nucl. Mat. 337-339, 548 (2005).

5. H. S. McLean, B. I. Cohen, D. N. Hill, E. B. Hooper, J. M. Moller, C. Romero-Talamás, and R. D. Wood, these proceedings.

6. B. W. Stallard, E. B. Hooper, S. Woodruff, R. H. Bulmer, D. N. Hill, H. S. McLean, R. D. Wood, and the SSPX Team, Phys. Plasmas 10, 2912 (2003).

7. C. R. Sovinec, B. I. Cohen, G. A. Cone, E. B. Hooper, and H. S. McLean, Phys. Rev. Letters 94, 035003 (2005).

8. B. I. Cohen, E. B. Hooper, R. H. Cohen, D. N. Hill, H. S. McLean, R. D. Wood, and S. Woodruff, Phys. Plasmas 12, 056106 (2005).

9. E. B. Hooper, T. A. Kopriva, B. I. Cohen, D. N. Hill, H. S. McLean, R. D. Wood, and S. Woodruff, Phys. Plasmas 12, 092503 (2005).

10. T. R. Jarboe, Plas. Phys. Control. Fusion 36, 945 (1994).

11. S. Woodruff, D. N. Hill, B. W. Stallard, R. Bulmer, B. Cohen, C.T. Holcomb, E. B. Hooper, H. S. McLean, J. Moller, R. D. Wood, Phys. Rev. Letters 90, 095001 (2003).

12. D. D. Ryutov, R. H. Cohen, and D. N. Hill, Plasma Phys. Reports 29, 605 (2003).

13. C. A. Romero-Talamás, E. B. Hooper, D. N. Hill, B. I. Cohen, H. S. McLean, R. D. Wood, and J. M. Moller, poster at the ICC Workshop, Austin TX, Feb. 13-16, 2006.

14. S. Woodruff, B. W. Stallard, H. S. McLean, E. B. Hooper, R. Bulmer, B. I. Cohen, D. N. Hill, C. T. Holcomb, J. Moller, and R. D. Wood, Phys. Rev. Letters 93, 205002 (2004).

15. S. Woodruff, B. I. Cohen. E. B. Hooper, H. S. McLean, B. W. Stallard, D. N. Hill, C. T. Holcomb, C. Romero-Talamás, R. D. Wood, G. Cone, and C. R. Sovinec, Phys. Plasmas 12, 052502 (2005). 


\section{Figure Captions}

Fig. 1. Typical SSPX discharges. (Left) Pulse formation: A spheromak formed by a high current pulse transitions to the confinement regime with $\lambda_{\text {gun }} \leq 10 \mathrm{~m}^{-1}$. (Right) Steadilybuilding: A spheromak is formed with $\lambda_{\text {gun }}>10 \mathrm{~m}^{-1}$ throughout the discharge. The bias poloidal flux is less in the second discharge than the first.

Fig. 2. Poloidal flux amplification in simulations of a steadily driven spheromak.

Fig. 3. (Left) Startup of the pulse-formation discharge in Fig. 2. (Right) Startup simulation; the gun current is identical to that in the experiment.

Fig. 4. Effects of voltage spikes (upper-left) due to reconnection events. (Upper-right) Increase in toroidally-averaged, poloidal field at each event. (Lower left) Energy in the MHD modes driven by the current column from the gun. (lower right) $T_{e}$ changes associated with each event.

Fig. 5. Magnetic field line Poincare puncture plots at the indicated times in Fig. 4. The first plot is during the voltage fall after the first pulse, and the second is at the peak of the second.

Fig. 6. Current sheet generated by the $n=1$ mode on the gun-current column along the geometric axis. 

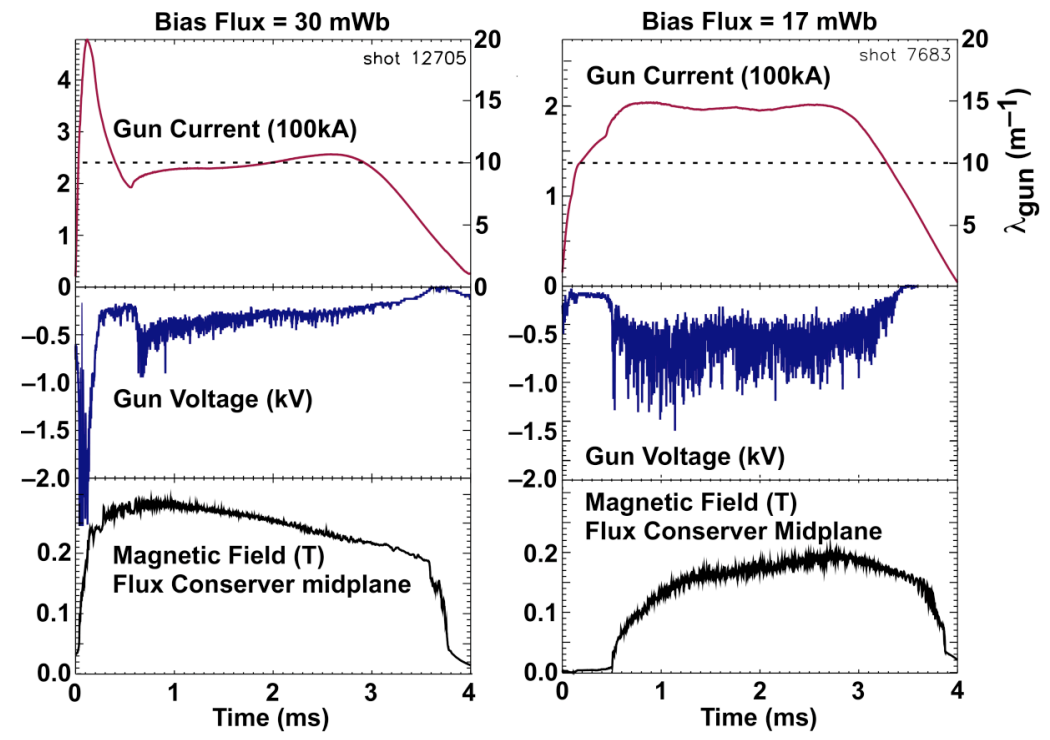

Hooper, Fig. 1 


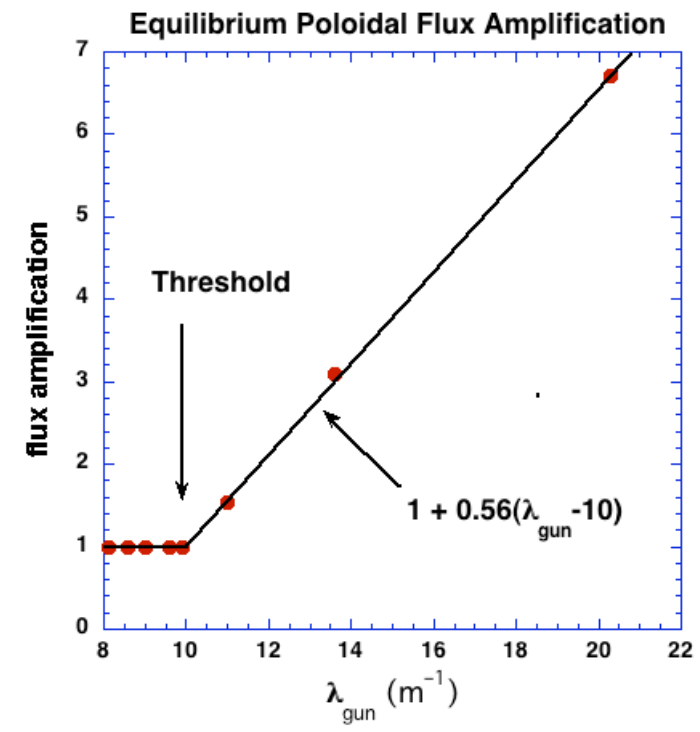

Hooper, Fig. 2 


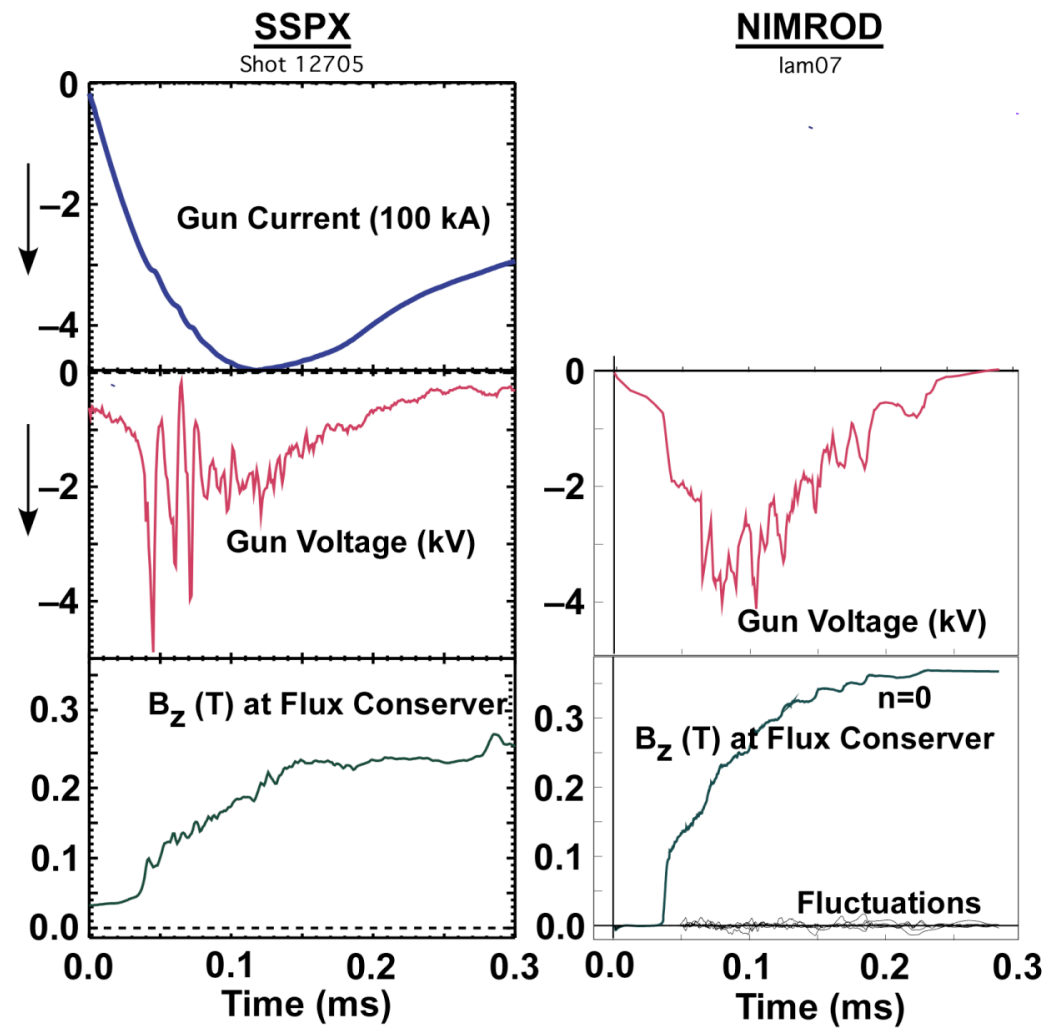

Hooper, Fig. 3 

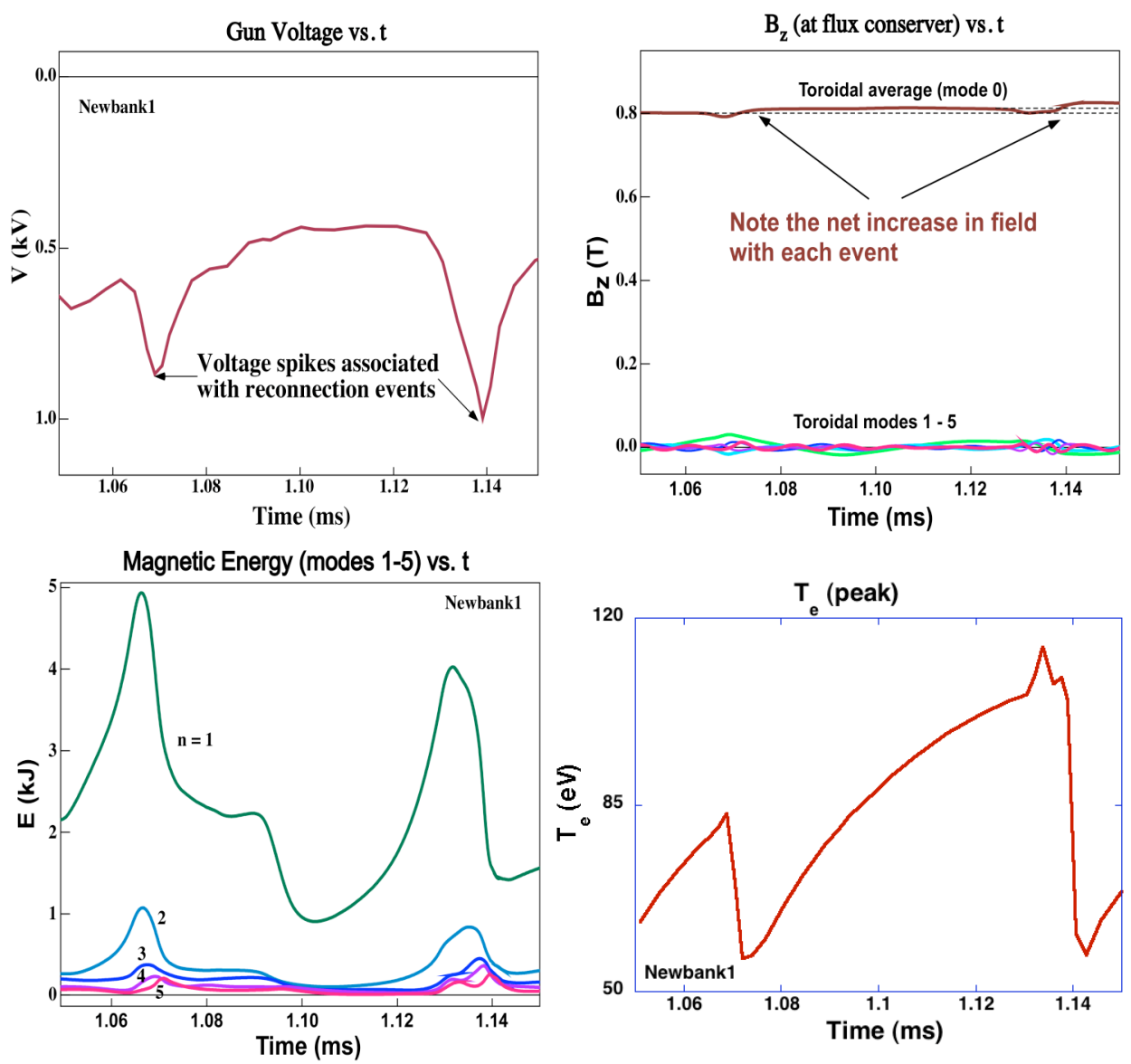

Hooper, Fig. 4 

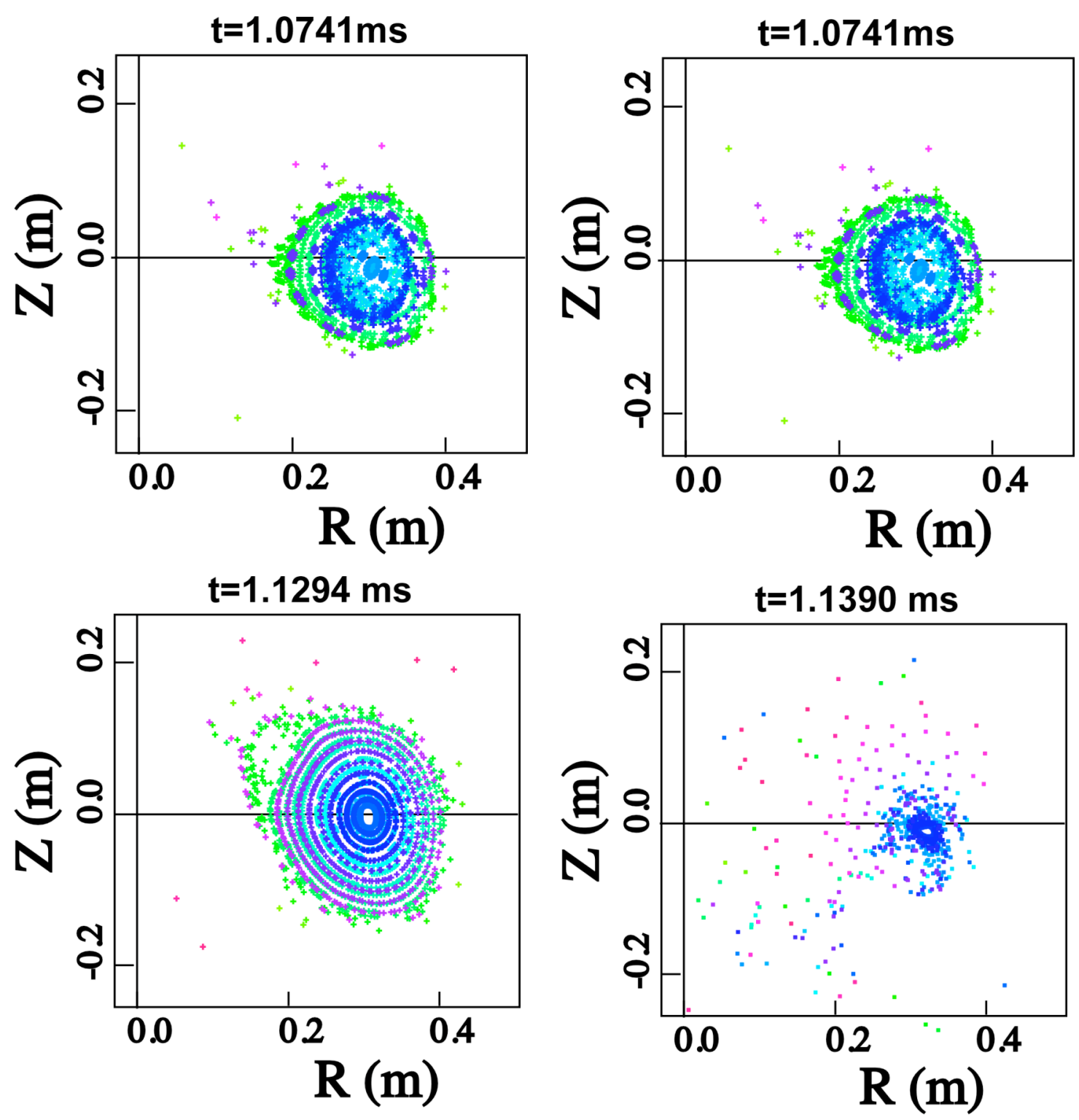

Hooper, Fig. 5 


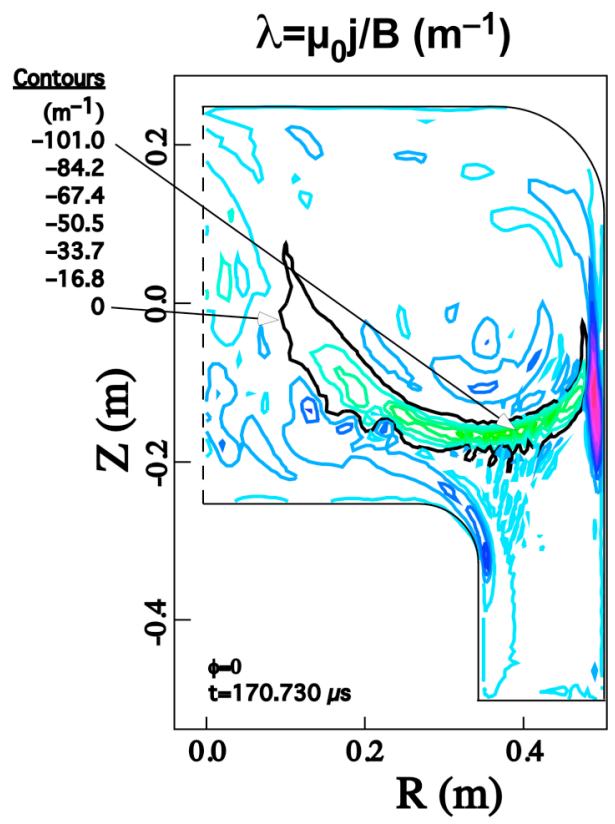

Hooper, Fig. 6 